\title{
Simultaneous Large Bi-Atrial Device-Related Thrombi
}

Jonas Dominik Häner, MD and Christian Seiler, MD

Department of Cardiology, Inselspital, University of Bern Hospital, Bern, Switzerland (J.D.H., C.S.)

Address for correspondence:

Jonas Dominik Häner, MD

Inselspital, University Hospital of Bern

3010 Bern, Switzerland

Phone: +4131632 9654, Fax: +41316324299

E-mail: jonas.haener@insel.ch

30-words-abstract: Simultaneous appearance of a pacemaker-lead associated thrombus and a left atrial appendage closure device related thrombus in a patient with urothelial carcinoma shown in a transesophageal echocardiography

Key words: intra-cardiac thrombus; device-associated thrombus; left atrial appendage closure; transesophageal echocardiography; paraneoplastic hypercoagulability

Acknowledgments: We thank Raphael Grossenbacher for the acquisition of the 3Dtransesophageal echocardiography images. 


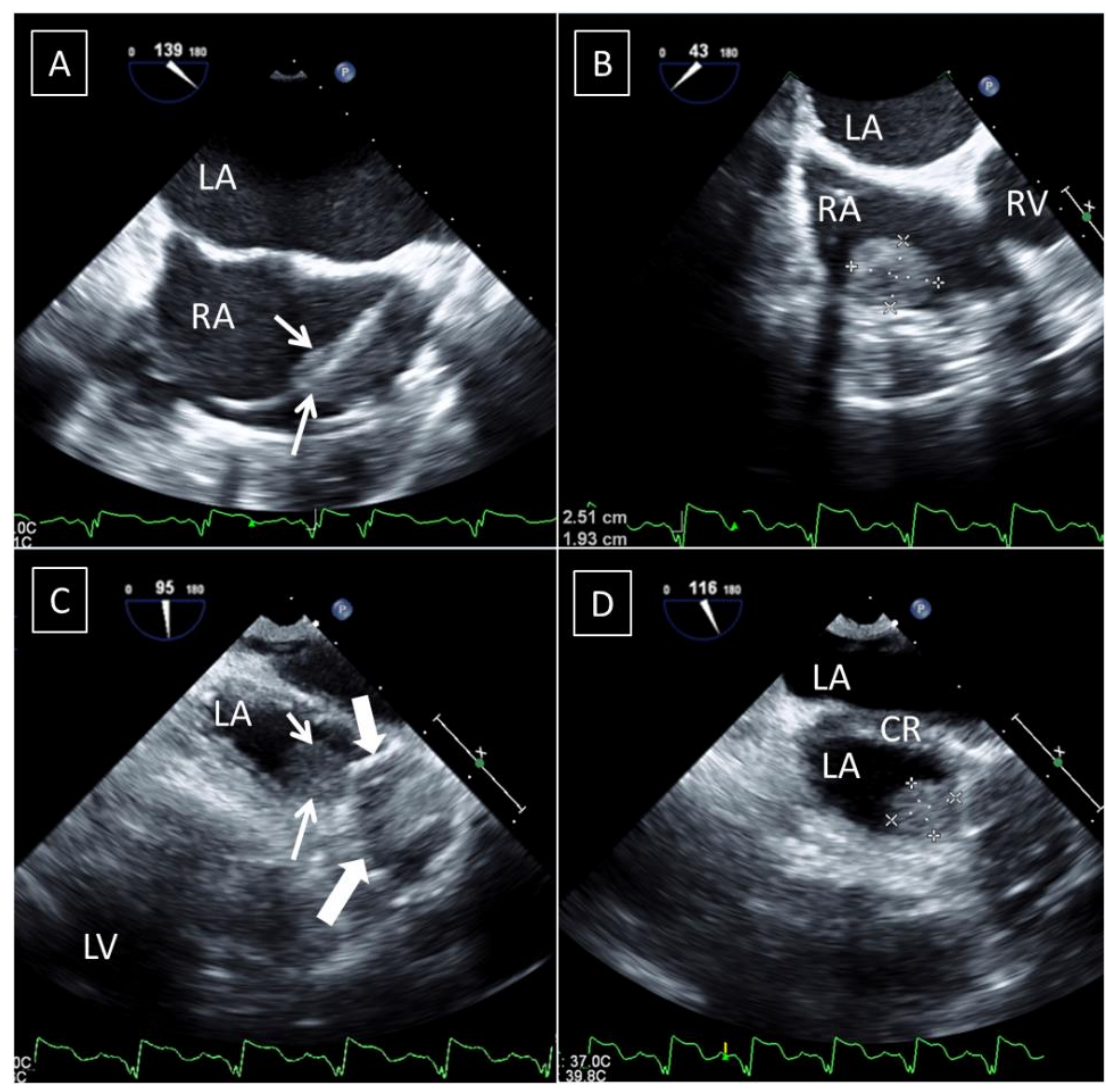

figure 1

A 84-year-old male patient underwent routine transesophageal echocardiography five months after closure of the left atrial appendage (LAA) using a Watchman ${ }^{\mathrm{TM}}$-Device as an alternative stroke prevention strategy in atrial fibrillation and following several bleeding complications under oral anticoagulation from an urothelial carcinoma. Immediately after closure of the LAA oral anticoagulation had been stopped and the patient remained under acetylic salicylic acid. Besides paroxysmal atrial fibrillation, he had a history of coronary artery disease with coronary artery bypass grafting 18 years and myocardial infarction ten years ago and a moderate aortic stenosis. Five months before, a three-chamber pacemaker was implanted for cardiac resynchronization therapy (CRT) for severely reduced systolic left ventricular function and left bundle branch block. The actual transesophageal echocardiography revealed two intra-cardiac thrombi. The larger thrombus was attached to the right ventricular CRT-lead and situated in the right atrium (figure 1, panels $A$ and $B$ : arrows pointing at pacemaker lead associated thrombus, $25 \times 19$ mm; LA: left atrium, RA: 
right atrium; RV: right ventricle). The second thrombus adhered to the LAA closure device in the left atrium (figure 1, panels $C$ and D: thin arrows showing thrombus, $9 \times 10 \mathrm{~mm}$; bold arrows indicating LAA closure device; LV: left ventricle, CR: coumarine ridge). A patent foramen ovale was excluded. The simultaneous appearance of two thrombi in the left and the right atrium was assumed to be caused by increased, possibly paraneoplastic, hypercoagulable conditions in this patient suffering from urothelial carcinoma [1]. A large meta-analysis underlined, that in patients with cancer-associated thrombosis, low-molecular weight heparins $(\mathrm{LMWH})$ reduce the recurrence of venous thromboembolism (VTE) as compared to vitamin-K antagonists, whereas direct oral anticoagulants (DOAC) do not [2]. However, a very recently published cohort study including 200 patients with cancerassociated VTE treated with rivaroxaban has suggested preserved safety and efficacy, but this study lacked of a control group and outcomes were compared with past-published data on LMWHs [3]. Still, actual recommendations on DOACs advise caution when used in cancer patients [4]. As evidence for the treatment of device-related thrombosis other than central venous catheter-associated thrombosis in patients with cancer is limited, the presented patient was referred to the regional hospital for the initiation of an anticoagulation using (low-molecular weight) heparins, in accordance to current recommendations on the treatment of cancer-associated VTE [5-6].

\section{Compliance with Ethical Standards}

Conflict of Interest: The authors declare that they have no conflict of interest.

Ethical approval: This article does not contain any studies with human participants performed by any of the authors.

Informed consent: General informed consent for retrospective analyzes was obtained from the presented patient. 


\section{References}

1. Wun T, White RH (2009) Epidemiology of cancer-related venous thromboembolism. Best Pract Res Clinc Haematol 22:9-23. doi: 10.1016/j.beha.2008.12.001

2. Carrier M, Cameron C, Delluc A, Castellucci L, Khorana AA, Lee AY (2014) Efficacy and Safety of Anticoagulant Therapy for the Treatment of Acute Cancer-Associated Thrombosis: A Systematic Review and Meta-Analysis. Thromb Res 134(6):1214-1219. doi: $10.1016 /$ j.thromres.2014.09.039

3. Mantha S, Laube E, Miao Y, Sarasohn DM, Parameswaran R, Stefanik S, Brar G, Samedy P, Wills J, Harnicar S, Soff GA (2016) Safe and effective use of rivaroxaban for treatment of cancer-associated venous thromboembolic disease: a prospective cohort study. ] Thromb Thrombolysis [Epub ahead of print 2016 Sep 30]. doi: $10.1007 / \mathrm{s} 11239-016-1429-1$

4. Becattini C, Agnelli G (2016) Treatment of Venous Thromboembolism With New Anticoagulant Agents. J Am Coll Cardiol 67(16):1941-1955. doi: 10.1016/j.jacc.2016.01.072

5. Farge $D$, Bounameaux $H$, Brenner $B$, Cajfinger $F$, Debourdeau $P$, Khorana $A A$, Pabinger I, Solymoss S, Douketis J, Kakkar A (2016) International clinical practice guidelines including guidance for direct oral anticoagulants in the treatment and prophylaxis of venous thromboembolism in patients with cancer. Lancet Oncol 17(10):e452-e466. doi: 10.1016/S1470-2045(16)30369-2

6. Khorana AA, Carrier M, Garcia DA, Lee AY (2016) Guidance for the prevention and treatment of cancer-associated venous thromboembolism. J Thromb Thrombolysis 41(1):81-91. doi: 10.1007/s11239-015-1313-4 
Additional material (for possible online publication only)

1) 3D-TEE movie showing the pacemaker lead associated thrombus

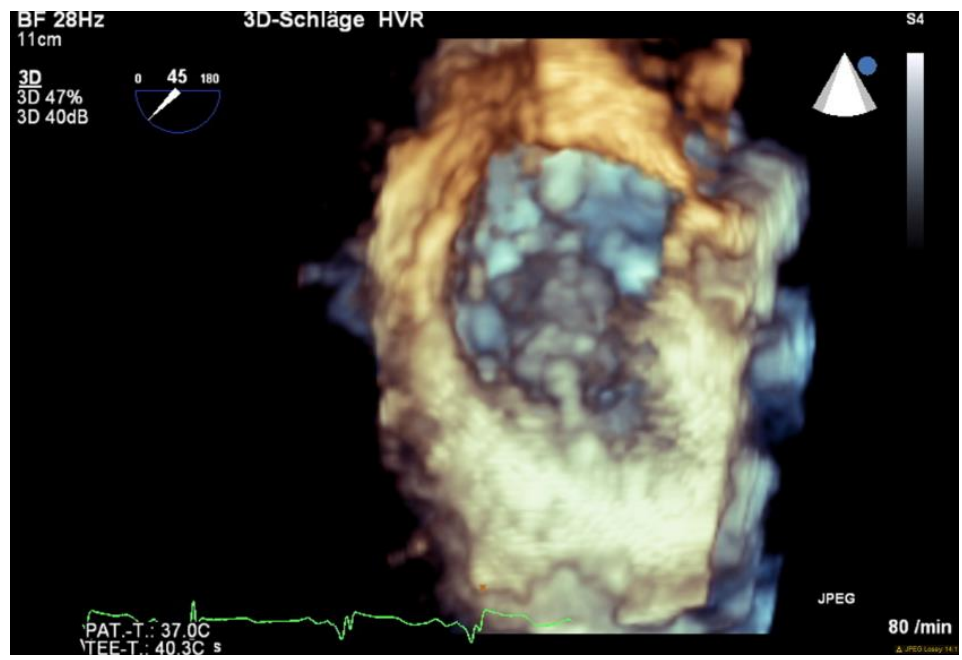

2) 2D-TEE movie of the pacemaker lead associated thrombus

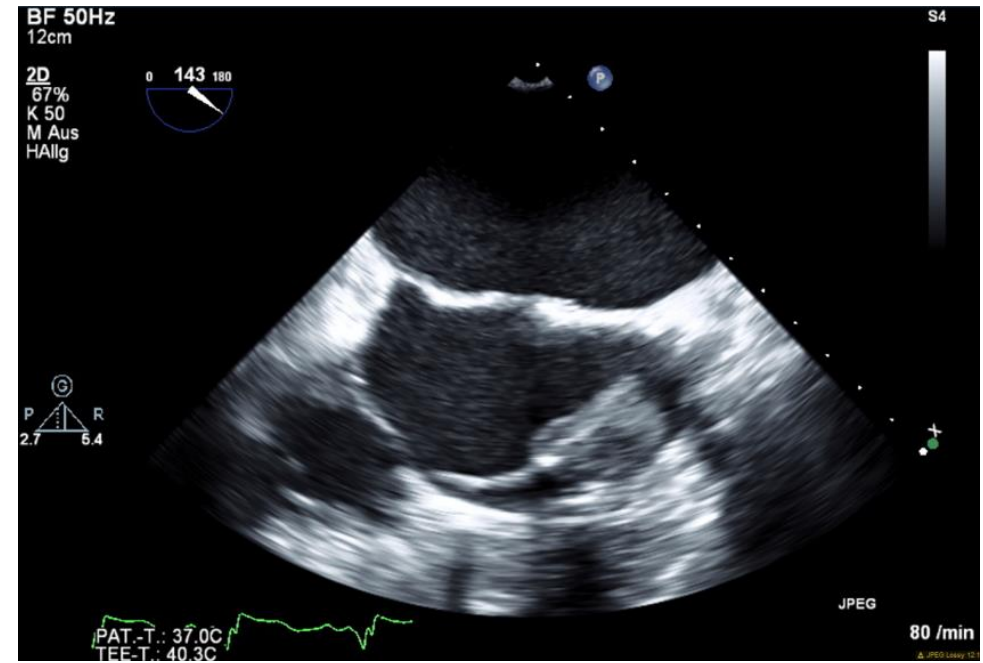

3) 2D-TEE movie of the LAA closure device thrombus

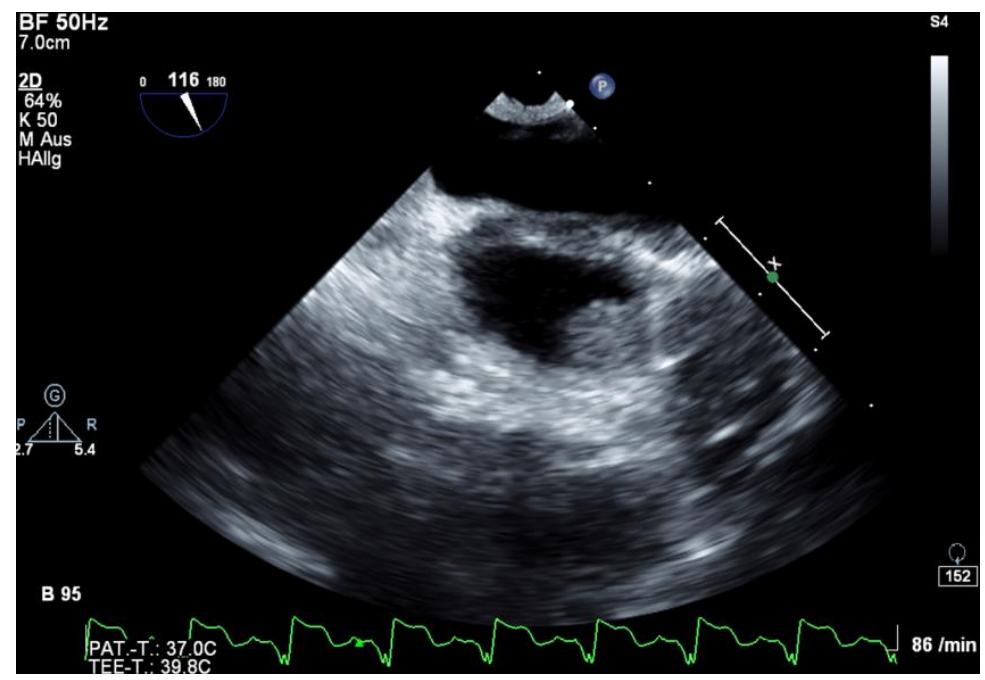

